Rabaska

Revue d'ethnologie de l'Amérique française

\title{
Les Archives acadiennes à l'Université du Maine à Fort-Kent
}

\section{Lisa Ornstein}

Volume 2, 2004

URI : https://id.erudit.org/iderudit/201704ar

DOI : https://doi.org/10.7202/201704ar

Aller au sommaire du numéro

Éditeur(s)

Société québécoise d'ethnologie

ISSN

1703-7433 (imprimé)

1916-7350 (numérique)

Découvrir la revue

Citer ce document

Ornstein, L. (2004). Les Archives acadiennes à l'Université du Maine à

Fort-Kent. Rabaska, 2, 325-327. https://doi.org/10.7202/201704ar d'utilisation que vous pouvez consulter en ligne.

https://apropos.erudit.org/fr/usagers/politique-dutilisation/ 


\section{Les Archives acadiennes}

à l'Université du Maine à Fort-Kent

23, promenade University

Fort Kent, ME 04743
Téléphone : (207) 834-7535

Télécopieur : (207) 834-7518

Courriel : acadian@maine.edu Toile :www.umflc.maine@edu/archives

\section{Origine}

La vallée du Haut-Saint-Jean se situe à la frontière commune de l'Acadie, du Québec et des États-Unis. Ce « Madawaska historique », colonisé à partir de 1785 par des Acadiens et des « Canadiens », est partagé depuis 1842 entre le Québec et le Nouveau-Brunswick au Canada et le Maine aux États-Unis. Elle représente une population d'environ 50000 personnes (19 000 au Maine et 31000 au Nouveau-Brunswick et Québec) dont la majorité retrace ses racines en Acadie et au Québec.

Malgré les pressions associées avec leur intégration à la culture angloaméricaine, les descendants américains des Madawaskayens ont su perpétuer une identité culturelle riche et spécifique et ils en sont fiers. Dans « la Vallée » (pour employer l'expression locale) l'on retrouve de nombreux exemples remarquables d'initiatives privées visant à la connaissance du passé ainsi qu'à la sauvegarde des traditions et des valeurs vivantes. Mais il a fallu attendre jusqu'à la fin des années 1980 pour la création de véritables archives régionales permettant la conservation et la mise en valeur de ce riche patrimoine.

En 1989, au cours de la première séance ordinaire, la $114^{e}$ Assemblée législative de l'état du Maine allouait des crédits budgétaires afin d'établir et de maintenir des Archives acadiennes à l'Université du Maine à Fort-Kent. Parmi les sept campus constituant le réseau de l'Université du Maine, l'Université du Maine à Fort-Kent était à l'origine le « Madawaska Training School », fondé en 1878 dans le but de former des enseignants bilingues pour la population francophone de la partie nord du comté d'Aroostook. Depuis, cette école a progressé et s'est transformée en une université moderne régionale, centrée sur les arts libéraux et offrant le baccalauréat et des diplômes associés aux étudiants réguliers et non réguliers, dont bon nombre n'auraient peut-être pas autrement accès aux avantages éducatifs qu'offre l'Université. 


\section{Mandat}

La mission des Archives acadiennes / Acadian Archives de l'Université du Maine à Fort-Kent porte sur les valeurs culturelles, le mode de vie et l'histoire de la population de la vallée du Haut-Saint-Jean. Les Archives sont au service de la communauté universitaire locale, de la population en général et des organismes, tant au plan régional que national et international et elles fournissent des services bilingues sur place. Ses fonctions se divisent en quatre volets : documentation, préservation, mise en valeur et diffusion.

1. Les Archives constituent un centre de conservation d'archives sonores et visuelles, de transcription d'enquêtes et de collections de manuscrits portant sur le folklore et le patrimoine vivant de la vallée du Haut-Saint-Jean. Elles recueillent également des documents portant sur l'histoire et la généalogie de la région. En plus, les Archives possèdent une collection de livres et d'imprimés (livres rares ou épuisés ainsi que des publications spécialisées et des ouvrages de référence) pour consultation sur place.

2. Elles sont un centre d'information qui offre sur place des services de consultation de ses collections ainsi que des renseignements sur le folklore, l'histoire, la généalogie et le patrimoine vivant de la région.

3. Elles forment en plus un centre de recherche qui réalise ses propres projets, rassemble de la documentation et produit des études et des publications (inventaires, études, documents audio-visuels, etc.) sur le patrimoine vivant et l'histoire orale. Les Archives produisent également des outils qui contribuent au développement de la recherche en histoire régionale.

4. Les Archives se veulent finalement un centre d'animation sur le patrimoine vivant et l'histoire régionale. Elles organisent des activités communautaires et des événements culturels qui célèbrent et mettent en valeur le patrimoine vivant et l'histoire régionale (expositions, concerts, ateliers, festivals, émissions radiophoniques ou télévisées, etc.). En outre, les Archives appuient les initiatives locales, en fournissant des conseils de spécialistes et de l'aide technique : services de consultation sur place, répertoires de personnes-ressources, outils pédagogiques, ateliers pédagogiques, prêts d'équipement, etc.

\section{Organisation et collections}

Depuis 1990, les Archives acadiennes occupent trois petits locaux à l'intérieur de la bibliothèque de l'Université du Maine à Fort-Kent. Il y a une salle de collections, un laboratoire audiovisuel ainsi qu'une salle polyvalente servant à la fois de bureaux pour les permanents et de salle de visiteurs. Bien que ses dimensions physiques soient assez restreintes, les Archives acadiennes sont bien équipées pour fournir sur place des services professionnels de documentation, de conservation et de mise en valeur. 
Les fonds des Archives acadiennes comptent plus de 30 pieds linéaires de documents, 7000 images photographiques, 450 enregistrements audiovisuels, 200 bobines de microfilms, 1850 revues et livres de référence, 110 cartes et d'autres matériaux portant sur l'histoire et la culture régionales. Notre catalogue informatisé est accessible par la toile et notre site offre des renseignements sur nos fonds et nos services tout en servant de point de contact pour des chercheurs et chercheuses à distance. Le personnel compte deux permanents aidés à l'occasion par des bénévoles ou des étudiants. Nous répondons à environ 1800 à 2100 demandes d'aide à la recherche par année.

La création des véritables archives est un travail complexe qui demande une planification solide et rigoureuse. Heureusement, les Archives acadiennes de l'Université du Maine à Fort-Kent bénéficient depuis le début de l'appui d'un conseil consultatif qui réunit des membres provenant de tous les coins de la région. En plus, Ronald Labelle, folkloriste au Centre d'études acadiennes à l'Université de Moncton, a suivi la mise sur pied des Archives en tant que conseiller principal et continue de fournir régulièrement de précieux conseils. Le travail de planification des Archives a été accompli pendant l'année 1990 par Lisa Ornstein et Guy Dubay. Depuis juillet 1991, $\mathbf{M}^{\text {me }}$ Ornstein et Nicholas Hawes, aidés à l'occasion d'étudiants, de contractuels et de bénévoles, assurent la permanence au sein des Archives.

LISA ORNSTEIN 